$\xi=-1$

\title{
Energy and Delay Constrained Packet Transmission MAC Protocol for Wireless Sensor Networks
}

\author{
Jin Young Lee,Seong Cheol Kim*,Hye Yun Kim \\ ${ }^{1}$ SK holdings, SangMyung University, SangMyung University \\ *Corresponding authorE-mail : jylee00@sk.com, sckim@smu.ac.kr,hykim@smu.ac.kr
}

\begin{abstract}
In this paper, we present an energy and time constrained packet transmission MAC Protocol which we call ED-MAC for event-driven applications such as fire detection in wireless sensor networks (WSNs). In this kind of WSNs, sensor nodes send a burst data when there is an event in the measuring field. These events are usually critical, so the measured burst data should be transmitted in limited time to their final destination node, sink node. Therefore, the packet transmission delay is considered to be a crucial requirement in event-driven applications. The ED-MAC protocol reduces the packet transmission delay and average node energy consumption in comparison to existing related MAC protocols.
\end{abstract}

Keywords:QoS, Multiple Access Control, Wireless Sensor Networks.

\section{Introduction}

In the event-driven applications of Wireless Sensor Networks (WSNs) such as object appearance, wild animal monitoring, and fire monitoring, burst data may be generated when such an event occurs. Furthermore, the measured data should be sent to the sink node in a predefined time. But normal measured data may be generated when no event occurs. Many research works have been done to resolve this problem [1][2][3][4][5]. In RMAC [2], all source nodes always send their packets at the beginning of a Sleep period. So packets collision at the beginning of the Sleep period may occurs if more than two nodes send PION frame. To solve this problem DW-MAC (Demand Wakeup MAC) [3] and SRMAC (Synchronous duty cycling MAC) [4] was proposed. Using the mapping function, DW-MAC resolves the packet collision problem in RMAC. But the DW-MAC protocol allows nodes to send only one packet during the Sleep period. The SR-MAC uses a scheduling mechanism that reserves few time slots in one Sleep period. It allows the nodes to transmit multiple data packet in an operational cycle. As existing approaches are mainly optimized for energy consumption of the node, we found that they become less efficient in transmission delay as burst data is generated.

\section{Design of the Ed-Mac Protocol}

The ED-MAC (Energy and Delay constrained packet transmission $\mathrm{MAC}$ ) is a synchronous duty-cycle MAC protocol. In ED-MAC one operation period are divided by 4 parts; Sync1 period, Data period, Sync2 period, and Sleep period. All sensor nodes in EDMAC wake up at the beginning of the SYNC1 period and send special control packet periodically to synchronize with their neighbor nodes. During the Data period sender nodes send control frames to their neighbor nodes. Here we call the control frame as the slot-reserved and operational cycle expansion informationinformed frame, SIF (Slot Information Frame) frame. In addition to the sender address, receiver address, duration of the transmission as in RTS/CTS (Request To Send/Clear To Send), a SIF frame also includes the number of slots, number of packets. In ED-MAC, Data period is divided into M slots. The Sleep period is also divided into M slots. But the Sleep period can be composed of several frames with $\mathrm{M}$ slots. The number of frames in Sleep period depends on the packets by sender node. ED-MAC adopts a flexible operational cycle according to the data traffic. Any node which sends its SIF frame first in Data period, then the node can send its data packet at the beginning of frame. During the Sync2 period all nodes wake up again to get the slot reservation and the operational cycle expansion information from a sink node. The Fig. 1 shows an example of the operation of ED-MAC. A sender node that firstly sent the SIF frame can send its data packet at the beginning of the Sleep period of each frame. So several unallocated slots may be exited at the end of the Data period.

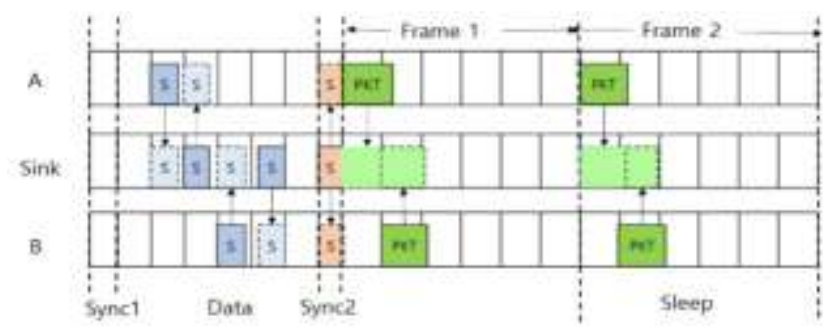

Figure 1:. ED-MAC Protocol scheduling for packet transmission.

\section{Performance Evaluation}

Table 1 shows the networking and energy parameters of the sensor node, which are used in the evaluation. We assumed here that all sender nodes are located in one hop away from the sink node. The network is composed of 39 sensor nodes and one sink node. And all the data packets are routed and destined to the sink node located at the center of the network structure. 
Table 1. Network Parameters

\begin{tabular}{|l|l|c|l|}
\hline Parameter & Value & Parameter & Value \\
\hline Bandwidth & $20 \mathrm{Kbps}$ & Data pkt size & 50 Bytes \\
\hline$T_{x}$ power & $0.5 \mathrm{~W}$ & $T_{\text {sync1 }}$ & $55.2 \mathrm{~ms}$ \\
\hline$R_{x}$ Power & $0.5 \mathrm{~W}$ & $T_{\text {sync } 2}$ & $10 \mathrm{~ms}$ \\
\hline Idle Power & $0.45 \mathrm{~W}$ & $T_{\text {data }}$ & $142.0 \mathrm{~ms}$ \\
\hline Sleep Power & $0.05 \mathrm{~W}$ & $T_{\text {sleep }}$ & $426.0 \mathrm{~ms}$ \\
\hline SIFS & $5 \mathrm{~ms}$ & PION & 14 Bytes \\
\hline DIFS & $10 \mathrm{~ms}$ & SRF, SIF & 14 Bytes \\
\hline RTS,CTS,ACK & $10 \mathrm{Bytes}$ & & \\
\hline
\end{tabular}

For easy comparison purpose it is assumed that one frame in a Sleep period is composed of 8 slots and a frame can be extended by 3 in maximum. And it is assumed that a node can generate three to sixteen data packets when an event occurs. Fig. 2 shows the average packet transmission delay of all protocols with number of data packet generated from node. As shown in the figure the ED-MAC protocol outperforms other related protocols in packet transmission delay between sensor nodes and sink node.

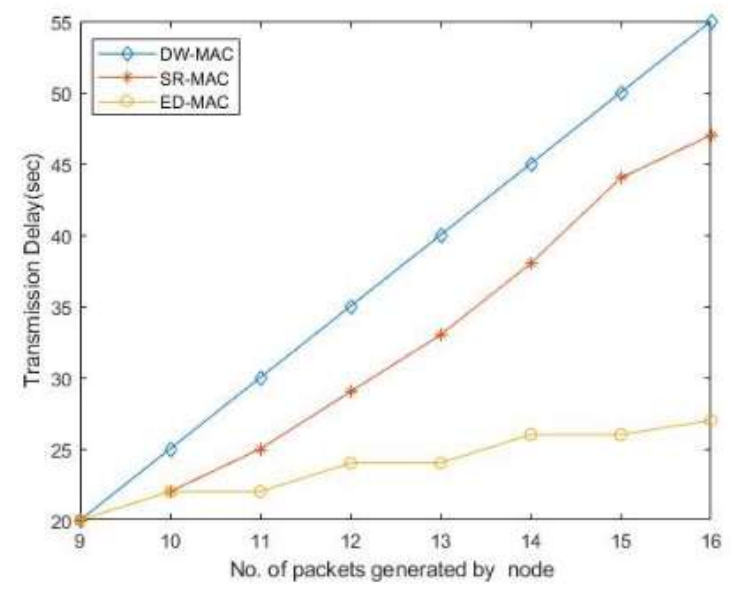

Figure 2:. Comparison of average packet transmission delay

Because DW-MACcannot transmit all data packet in a single operational cycle when number of packets exceeds 6 , the overall average transmission time increases. As the number of packets generated by node increases, the ED-MAC protocol shows better performance.Comparison of average node energy consumption with number of data packet generated from node is shown in the Fig. 3. The ED-MAC also outperforms other related protocols in average energy consumption.

\section{Conclusion}

MAC protocol for the event driven applications such as fire detection, wild animal appearance, and object tracking in WSNs. The proposed MAC protocol has a duty cycling scheme to reduce energy consumption due to idle listening of the node. In ED-MAC protocol, during the Sync2 period all nodes wake up again to get the slot reservation and the operational cycle expansion information from a sink node. The Sync2 period is used for operational cycle expansion when an event occurs. Compared other related MAC protocols, the ED-MAC protocol reduces the packet transmission delay and average node energy consumption in comparison to existing related MAC protocols, especially as number of packets by node increase.

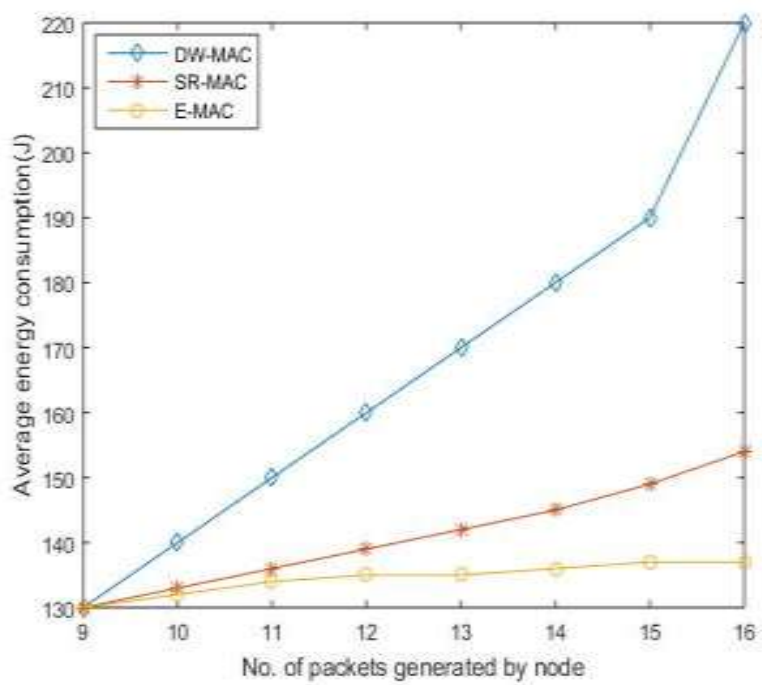

Figure 3:. Comparison of average node energy consumption

\section{References}

[1] W. Ye, J. Heidemann, and D. Estrin, An Energy-Efficient MAC Protocol for Wireless Sensor Networks, Proceedings of the 21st Annual Joint Conference of the IEEE Computer and Communications Societies, New York, NY (2002), Vol. 3, pp. 1567-1576.

[2] S. Du, A. Saha and D. Johnson, RMAC: a routing-enhanced dutycycle MAC protocol for wireless sensor networks, Proceedings of the 26th Annual IEEE Conference on Computer Communications (INFOCOM 2007) Barcelona, Spain (2007), pp.1478-1486.

[3] Y. Sun, S. Du, O. Gurewitz and D. Johnson, DW-MAC: a low latency, energy efficient demand wakeup MAC protocol for wireless sensor networks, Proceedings of the Ninth ACM International Symposium on Mobile Ad Hoc Networking and Computing (MobiHoc'08) (2008), pp. 53-62.

[4] H. Tang, J. Cao, X. Liu, and C. Sun, SR-MAC: A Low Latency MAC Protocol for Multi-Packet Transmission in Wireless Sensor Networks, Journal of Computer Science and Technology (2013), Vol. 28, No. 2, pp. 329-342.

[5] H. Y. Kim, S. C. Kim, A Delay Efficient and Burst Traffics Friendly MAC Protocol in Wireless Sensor Networks, Journal of Korea Multimedia Society (2017), Vol. 20, No. 2, pp. 254-260 